\title{
Inovação tecnológica e as questões reflexivas do campo da biossegurança
}

\author{
MARLI B. M. DE ALBUQUERQUE NAVARRO', \\ TELMA ABDALLA DE OLIVEIRA CARDOSO II, \\ NERT CUNHA VITAL ${ }^{I I I}$ \\ e BERNARDo ELIAS CORREA SOARES ${ }^{I V}$
}

\section{Inovação em saúde}

$\Delta$ SOCIEDADE industrial construiu um arcabouço ideológico que legitimou a concretização do acúmulo de conhecimentos e de tecnologias impactantes como meios para viabilizar um mundo mais igualitário, confortável e seguro. Esses meios, fundamentados na ciência e na tecnologia (sem a valorização e observância da perspectiva da precaução), seriam capazes de prover o mundo de abundância, diminuindo e/ou controlando a escassez, a fome, as calamidades naturais, as pandemias etc. Considerava-se que os problemas econômicos eram exclusivos das ciências econômicas, os problemas de saúde pública próprios das ciências da saúde, os problemas sociais específicos das ciências sociais e das iniciativas assistencialistas para consolidar a modernidade e administrar os riscos. Entretanto, com a "globalização" caracteriza-se como desafio a formulação do pensar baseado na complexidade, considerando as múltiplas vertentes que configuram as realidades dinâmicas e integradas de processos definidores do desenvolvimento (Navarro; Cardoso, 2005).

O processo tecnológico e suas inovações apresentam-se para a sociedade como fator paradoxal. Ele gera expectativas positivas e negativas, incertezas e desafios, como fatores demonstrativos das constantes construções e reconstruções das noções de risco, a partir do impacto informacional e do contexto social e conjuntural que envolve determinado risco.

A dinâmica dos processos tecnológicos atuais está ancorada na perspectiva do tríplice diálogo entre saber científico, interesses da indústria e do mercado, voltados para otimização do lucro por meio da capacidade do potencial de inovação.

Sublinha-se que a inovação tecnológica compreende a avaliação, a seleção e o fomento de iniciativas representativas do conhecimento científico de grande potencial de uso social e enquanto produto que alimenta o processo tecnológico de mercado, onde circulam projetos que hoje envolvem, por exemplo, 
biotecnologia, nanotecnologia, clonagem, uso de células tronco etc. Esses projetos requerem a participação efetiva da biossegurança como área avaliadora do potencial de risco ao ambiente e à saúde pública (abrangendo a saúde humana e animal), dos instrumentos fiscalizatórios e das práticas laboratoriais e sua relação com a segurança.

A complexidade que hoje está situada no campo da biossegurança envolve, sobretudo, a distribuição dos riscos. É exigido da sociedade um posicionamento constante no sentido da elaboração frequente de escolhas. As ações cotidianas, antes voluntárias e mais motivadas pelo desejo e pela afetividade, são substituídas por processos cognitivos, que devem abalizar tais escolhas. Somos contemporâneos de uma variedade de riscos globais com importantes dimensões pessoais (Navarro; Cardoso, 2005). "Os artefatos e as práticas da biotecnociência são percebidos como uma espécie de Jano de duas faces: uma prometedora e outra ameaçadora e a percepção pública dessas práticas está determinada por crenças, visões de mundo e ideologias, muitas vezes incomensuráveis entre si e nem sempre legítimas para a sociedade como um todo" (Schramm, 2004, p.2).

\section{Biotecnologia}

Atualmente encontra-se à nossa disposição uma multiplicidade de tecnologias que permitem, por exemplo, alterações genéticas em animais de laboratório; questiona-se se tais tecnologias podem ser aplicadas também ao homem (Winnacker, 2000).

As biotecnologias, em geral, compreendem a utilização de agentes biológicos, animais e plantas e determinadas células humanas, objetivando a obtenção de processos, produtos e serviços.

Como qualquer nova tecnologia, os riscos e os impactos das biotecnologias são ainda pouco conhecidos, e, portanto, objeto de uma criteriosa análise de risco. Essas análises devem ser respaldadas por estudos de segurança e de impacto ambiental, quando cabível. Atualmente, ainda há pouco conhecimento das consequências e das técnicas de controle sobre as construções genéticas, após a inserção no genoma da célula hospedeira; portanto, as análises de risco devem considerar o enfrentamento das complexidades, vulnerabilidades e das incertezas. Diante desse quadro, vem sendo cada vez mais aplicado o princípio da precaução, como norteador das avaliações e decisões de problemas que possam envolver riscos ambientais e para a saúde pública. O princípio da precaução foi desenvolvido para responder a esses problemas, uma vez que envolvem limitações na capacidade de predição da ciência para questões práticas (Freitas, 2003). Esse princípio é uma das ferramentas essenciais da biossegurança na execução das análises de risco das novas biotecnologias.

A partir dos resultados processados nos laboratórios, verificou-se uma corrida para a abertura de empresas comerciais, no geral subsidiárias de grandes universidades e centros hospitalares de pesquisa (Castells, 1999). 
A Genentech (Universidade de São Francisco), a Cetus (Universidade de Berkeley) e a Biogen (Universidade de Cambridge) constam como as primeiras empresas a usar as novas tecnologias genéticas para aplicações na medicina. Tais inovações foram logo depois seguidas pelo setor agroindustrial. Contudo, desse processo tecnológico, enfrentaria algumas dificuldades, focalizadas, sobretudo, em questões de viabilidade técnica e obstáculos legais, localizados nos fundamentos da ética e da segurança. Estes fatores adiaram a expansão da chamada "revolução biotecnológica", durante a década de 1980. Neste contexto um considerável valor de investimentos de capital de risco foi perdido, e algumas gigantes farmacêuticas (Hofman - La Roche-Merck) adotaram como segurança de investimento a estratégia de absorver as pequenas empresas processadoras de inovações, juntamente com a competência técnica e científica de seus pesquisadores, visando garantir os benefícios comerciais das pesquisas biotecnológicas. Os resultados dos investimentos verificariam retornos entre o final dos anos 80 e toda a década de 1990, período que registrou a revitalização dos projetos biotecnológicos, com enfoque centrado na engenharia genética. (Castells, 1999, p.65)

O desenvolvimento biotecnológico observado desde então abrangeu com maior ênfase os interesses articulados entre a ciência, a viabilidade tecnológica e os produtos de grande lucratividade para as empresas voltadas para a inovação tecnológica. Esses investimentos se deram nos países desenvolvidos, por grandes empresas, focados pelo mercado, voltados para as doenças que neles predominam (medicamentos cardiovasculares e do sistema nervoso central respondem por cerca de $50 \%$ das vendas globais da indústria). Os países pobres e em desenvolvimento englobam $80 \%$ da população mundial, mas respondem por apenas 20\% das vendas mundiais de medicamentos (Médecins sans Frontières, 2001). Nesse sentido, cabe ainda apontar a discussão sobre investimentos, biotecnologia e benefícios sociais. Tal discussão está amplamente vinculada ao setor da saúde, centrada na questão das doenças negligenciadas e as políticas de inovação tecnológica.

O relatório da Comissão de Macroeconomia e Saúde da Organização Mundial da Saúde divide as doenças em três categorias distintas (Morel, 2005, p.39):

TIPO I: Doenças existentes em países ricos e pobres, com grande número de populações vulneráveis em todos eles (sarampo, diabetes, hepatite B);

TIPO II: Doenças existentes em países ricos e pobres, mas com muito maior prevalência nos últimos (HIV/Aids, tuberculose);

TIPO III: Doenças exclusivas ou majoritariamente prevalentes em países pobres (doença de Chagas, tripanossomíase africana ou doença do sono, oncocercose)

Ressalta-se que as doenças negligenciadas são classificadas como do Tipo III.

Esse relatório, ao abordar os investimentos em inovação tecnológica no setor da saúde, mostra que desde o início dos anos 1990, em termos globais, 
há um enorme déficit nas pesquisas relacionadas às doenças negligenciadas, também conhecidas como "doenças da pobreza" ou ainda "doenças tropicais". "Esta carência foi batizada como déficit 10/90 (10/90 gap), pois apenas 10\% do investimento anual em pesquisa em saúde são destinados às doenças que afligem $90 \%$ da população mundial" (Morel, 2005, p.39).

Até o início da década atual, os esforços em pesquisas e desenvolvimento tecnológico $(\mathrm{P} \& \mathrm{D})$ para doenças negligenciadas foram limitados a

poucas iniciativas para tuberculose e malária, apesar dos expressivos avanços científicos alcançados, até na área da biotecnologia e sequenciamento genético, na compreensão dos organismos responsáveis por muitas dessas doenças e que poderiam ser usados no desenvolvimento de novos medicamentos. Esbarram, contudo, no desinteresse das firmas privadas, cuja capacitação é importante principalmente nas fases de desenvolvimento, testes e registro de medicamentos. (Bastos, 2006, p.288)

Segundo a Drugs for Neglected Diseases Initiative (DNDi), organização de apoio às iniciativas para o desenvolvimento de fármacos para as doenças negligenciadas, há um grande volume de trabalhos científicos que tratam da biologia, imunologia e genética dos parasitas causadores dessas doenças, porém todo esse conhecimento não consegue se reverter em novas ferramentas terapêuticas para as pessoas afetadas. Ao contrário, tais doenças têm sido progressivamente marginalizadas por aqueles encarregados pelos programas de pesquisa tanto do setor público quanto do privado, essencialmente porque as pessoas que sofrem de doenças negligenciadas são pobres, e não oferecem um retorno lucrativo suficiente para que a indústria farmacêutica invista em pesquisa e desenvolvimento de novos medicamentos voltados para essas doenças. Fica claro, portanto, que a crise de falta de medicamentos para doenças negligenciadas não chegou às atuais proporções por falta de conhecimento científico, e nem somente pelo hiato entre a pesquisa básica e a pré-clínica. Essa crise é o resultado tanto das insuficientes políticas públicas voltadas para P\&D de medicamentos de interesse nacional dos países em desenvolvimento, quanto da falha de mercado, provocada pelo baixo interesse econômico que esses pacientes representam para a indústria (DNDi, 2004).

Diariamente, mais de 35.000 pessoas morrem de doenças infecciosas e negligenciadas como Aids, malária, tuberculose, assim como de leishmaniose, doença de Chagas e doença do sono. (Costa, 2007, p.1)

De um total de 1.393 novos fármacos aprovados nos últimos 25 anos, apenas $1 \%$ foi destinado a doenças tropicais. Em 2000, dos 137 medicamentos para doenças infecciosas componentes do pipeline da PhRMA (associação de firmas farmacêuticas norte-americanas), apenas um tinha como indicação a doença do sono e um outro, a malária. Esses dados são apresentados no apelo por mais P\&D, pela organização DNDi e Médicos Sem Fronteiras (Marques, 2002). 
Tal contexto também contribui enfaticamente para ampliação do campo reflexivo e das abordagens críticas contidas no campo da biossegurança, uma vez que ele é demonstrativo das questões complexas e indicadoras de percepções múltiplas da abrangência dos benefícios dos projetos biotecnológicos e de seu alcance social.

O sistema tecnológico sobre o qual estamos ancorados hoje surgiu nos anos 1970, devido à importância dos contextos históricos específicos dos investimentos tecnológicos dos países e do modo particular de interação entre a tecnologia e a sociedade, contexto em que a biossegurança surgiu como critério de segurança e passou a agregar um valor dialógico entre a ciência e a sociedade, ao favorecer os métodos para monitoramentos de riscos na elaboração e na aplicação das inovações tecnológicas, contribuindo também para subsidiar os debates sobre a expansão biotecnológica e situar a questão da segurança, dos custos e dos benefícios de tais processos inovadores.

A fluidez da informação, em razão do advento de novos meios de comunicação; a internacionalização da economia, em razão do advento dos blocos econômicos, das empresas transnacionais e dos organismos internacionais de fomento; e a desterritorialização das decisões políticas, motivadas pelo advento dos organismos multilaterais e das comunidades internacionais são interfaces que trazem consequências relevantes para a gestão dos recursos naturais globais e a disseminação de novas tecnologias, visto que as esferas de decisão estão cada vez mais nas mãos dos atores políticos globais, e cada vez mais distantes da sociedade civil organizada (movimentos sociais) dos Estados-nação e do Terceiro Setor (as ONG e Oscip).

Destaca-se a importância dos investimentos em inovação para qualquer Estado-nação que pleiteie um lugar de destaque entre as grandes potências mundiais, pois os agentes econômicos dos diversos países apenas atingem posição equilibrada de negociação se estiverem amparados por padrões compatíveis de acesso e desenvolvimento de novas tecnologias. Tecnologia, no mundo moderno, significa poder. No caso em apreço, biopoder, conceito elaborado por Michel Foucault para contextualizar a importância e a prática da regulação dos corpos, mediante diversas técnicas, a fim de tornar eficaz o controle de populações. Os Estados modernos, a partir da segunda metade do século XVIII, estruturaram os mecanismos capazes de caracterizar a "biopolítica" ou "biopoder" elaborando e legitimando um conjunto de processos de controle, tais como a proporção dos nascimentos e dos óbitos, a taxa de reprodução, a fecundidade, a longevidade e a mortalidade de uma população, relacionado esses dados com os problemas de ordem econômica e política (Foucault, 1999).

O que acontece, no contexto biotecnológico atual, é que os detentores do conhecimento, e, portanto, do poder, são as empresas transnacionais, que financiam as pesquisas e as descobertas, possuem os direitos sobre a propriedade intelectual destas e fundamentam a sua superioridade de maneira pragmática, 
sob os auspícios da técnica e do mecanicismo. Assim, a transgenia, como um dos processos biotecnológicos, traz em si a característica da funcionalidade da ciência, mas o seu caráter temporal e fluido não é facilmente identificável, o que fundamenta a utilização da tecnologia como uma "verdade inquestionável", uma "assertabilidade justificada".

A Revolução Biotecnológica tem como atores principais os conglomerados agroindustriais, farmacêuticos e alimentares, que determinam as "verdades" que lhes são convenientes, pois, ao financiar e dirigir as pesquisas, cerceiam a opinião pública mundial e desprezam o interesse coletivo, divulgando apenas os resultados das pesquisas que sejam favoráveis à nova tecnologia e satisfatórios aos interesses da economia de mercado.

\section{Biossegurança}

No contexto da inovação tecnológica, a biotecnologia aborda o potencial e a natureza das tecnologias, trazendo consigo a discussão sobre os impactos e os riscos dos produtos originários dessas tecnologias e de sua utilização.

Os processos relacionados exclusivamente à perspectiva de redução de custos, agilidade de produção, desenvolvimento de novos produtos visando garantia de maior lucratividade comercial, embora possam estar enquadrados nos conceitos de inovação tecnológica, podem gerar danos à saúde pública e ao ambiente.

Os avanços da biotecnologia e da biologia molecular colaboraram para a valorização da diversidade biológica, considerada tanto no âmbito dos ecossistemas como no da variabilidade das espécies e dos códigos genéticos, para a realização de pesquisas e desenvolvimento de tecnologias capazes de aperfeiçoar ou desenvolver processos industriais e produtos. Sob esse aspecto, "a biodiversidade é compreendida como um bem econômico estratégico: um manancial de 'conhecimentos' cristalizados em sistemas e estruturas complexas advindo de um longo processo evolutivo e cujo comprometimento representa uma perda incomensurável para a humanidade" (Cezar; Abrantes, 2003, p.226).

Diante das circunstâncias de incerteza científica, do volume de investimentos em biotecnologia realizado por grandes empresas, da necessidade de preservação ambiental e da saúde pública, a biossegurança consolida-se como uma área de importância pela sua capacidade multidisciplinar e dialógica, a fim de diminuir as inquietações da sociedade diante de das inovações de grande impacto e as possibilidades dos recursos de construtores de segurança dos processos científicos.

As relações entre inovações tecnológicas e as preocupações ambientais estariam impulsionando a adoção de mecanismos limpos, ambientalmente saudáveis, que devem ser compatíveis com as prioridades socioeconômicas, culturais e ambientais globalmente determinadas, contexto em que o princípio da precaução, como base para a biossegurança, consta como critério fundamental para viabilizar a aplicabilidade dos projetos. Sua popularização ocorreu recente- 
mente quando eclodiram crises sanitárias de grande repercussão, como a encefalopatia espongiforme bovina (mal da vaca louca) e os conflitos em torno dos organismos geneticamente modificados.

Nessa situação, a ampla compreensão do princípio precautório pelos atores sociais envolvidos influi sobre as decisões que minimizam conflitos e riscos. $\mathrm{O}$ exercício da precaução faz cair a barreira entre laboratórios e sociedade, experiência e experimentação, tornando os indivíduos ao mesmo tempo experimentadores e experimentados diante do suposto risco (Ewald, 2001). Pode-se definir o Princípio da Precaução como diretriz que busca regular a participação do conhecimento técnico e científico e o conhecimento de senso comum na previsão e no combate a degradações ambientais potencializadas por tecnologias.

A prerrogativa do incentivo voltado para a consolidação de políticas de biossegurança está colocada para os países como necessidade de formulação de critérios, métodos e ações de caráter precautório de âmbito global.

Em 1992, foi elaborada a Declaração do Rio de Janeiro, durante a Conferência das Nações Unidas sobre Meio Ambiente e Desenvolvimento (Cnumad), no Rio de Janeiro, também conhecida por Rio 92. Ela nasceu com o objetivo de estabelecer uma nova e justa parceria global mediante a criação de novos níveis de cooperação entre os Estados, a sociedade e os indivíduos, trabalhando pela criação de acordos internacionais que respeitem os interesses de todos e protejam a integridade do sistema global de meio ambiente e desenvolvimento. Ao todo são 27 princípios. Dentre esses está o princípio 15 estabelecendo a aplicação da abordagem precautória, a fim de proteger o meio ambiente, quando houver ameaça de dano sério ou irreversível, ou quando houver ausência de absoluta certeza científica.

Posteriormente a esse, vários acordos internacionais passaram a adotar definições semelhantes para o Princípio da Precaução, dentre esses a Convenção da Diversidade Biológica (CDB), ocorrida em Cartagena (Colômbia), em 2000. O Protocolo Internacional de Biossegurança, em harmonia com o Princípio da Prevenção, está incluído no contexto das recomendações da Convenção. Seu objetivo é contribuir para assegurar um nível adequado de proteção no domínio da transferência segura, manuseamento e utilização de organismos vivos modificados resultantes da biotecnologia moderna que possam ter efeitos adversos na conservação e utilização sustentável da diversidade biológica, tomando também em consideração os riscos para a saúde humana, e com particular enfoque no movimento transfronteiriço. Reconhece que o conhecimento científico sobre transgênicos é incompleto e permite que os países tomem medidas para prevenir danos ambientais na ausência de certeza científica sobre o dano, permitindo que as partes tomem a decisão de evitar ou minimizar tais efeitos potenciais adversos.

A Organização para Cooperação e Desenvolvimento Econômico (OCDE), organização internacional dos países comprometidos com a economia de livre mercado, também chamados "Grupo dos Ricos” pois agrega países que 
produzem mais da metade de toda a riqueza do mundo, em seu relatório "Riscos Emergentes no Século XXI", realça que

não somente a natureza dos riscos maiores parece estar mudando, mas também o contexto no qual estes aparecem e a capacidade da sociedade em gerenciá-los. A globalização sob todos os aspectos - econômico, tecnológico, cultural, ambiental - está crescendo rapidamente e ampliando a interdependência, de tal modo que vírus perigosos, poluentes e falhas técnicas tenham mais facilidade em se espalharem. De igual importância, as fronteiras da descoberta científica e da inovação tecnológica estão conhecendo expansão excepcionalmente rápida, a sociedade sendo assim confrontada com efeitos desconhecidos (e difíceis de conhecer), e, por conseguinte com escolhas extremamente difíceis. (OCDE, 2003, p.2)

Elas geram para a sociedade um clima de incertezas e desafios. Considerando que a mudança tecnológica em curso faz uso intensivo de processos biotecnológicos, que demanda conhecimentos científicos, a questão norteadora para a sociedade está relacionada aos níveis de exigência desses novos conhecimentos, assim como o modo como seriam feitas as readequações necessárias em um contexto de mudança. Por esse motivo a incorporação do Princípio da Precaução é importante. Esse Princípio foi definido durante a Declaração de Wingspread (Precautionary Principle Conference, 1998) como medidas a serem tomadas quando uma atividade gera ameaças de dano à saúde humana ou ao meio ambiente, mesmo se algumas relações de causa e efeito não forem completamente estabelecidas cientificamente.

Cabe, porém, ressaltar que o processo decisório inicia-se a partir de algo que é percebido como um problema. Atuam nesse processo o conhecimento disponível e um conjunto predeterminado de valores. Da composição desses elementos são geradas deliberações que culminam em uma ação prática e/ou em outro processo de decisão. O conhecimento disponível envolve a evidência empírica acumulada, as teorias e metodologias aceitas, informa sobre os riscos e possíveis benefícios associados a uma determinada decisão (Cezar; Abrantes, 2003).

Dada a natureza do conhecimento e da(s) metodologia(s) pressuposta(s), esse processo fornece, tipicamente, uma previsão probabilística. Os riscos e benefícios de uma dada decisão são, posteriormente, avaliados segundo um determinado padrão de valores. Após esse juízo sobre os riscos e benefícios envolvidos é que se opta por uma determinada linha de ação ou por um novo processo decisório. (Cezar; Abrantes, 2003, p.236)

O processo é, portanto, dinâmico, onde os cientistas reavaliam constantemente suas concepções e métodos, o que fornece maior confiabilidade às ciências.

\section{Considerações finais}

Os processos rápidos da aplicabilidade de novas tecnologias advindas da dinâmica da produção de novos conhecimentos científicos, que envolvem 
demandas de mercado, associadas aos interesses industriais, ao consumo e às expectativas sociais diante das inovações tecnológicas, vêm impondo ao universo acadêmico novos desafios na elaboração do conhecimento, principalmente naquele apoiado na perspectiva da complexidade com a concorrência de múltiplas áreas do saber. Considera-se, nesse contexto, a importância da solidariedade entre os saberes, para a compreensão dos fenômenos da natureza e da sociedade, como dinâmicas associadas para elaborar proposições e promover ações apresentadas como produtos, incluindo serviços e métodos.

A visualização da biossegurança e sua interface com as inovações tecnológicas e a bioética se estabelece pelas vertentes da política que se expressa por meio dos órgãos fiscalizadores.

O artigo 225 da Constituição brasileira identifica o meio ambiente ecologicamente equilibrado como bem de uso comum do povo e essencial à sadia qualidade de vida, impondo ao poder público e à coletividade o dever de defendê-lo e preservá-lo para as presentes e futuras gerações, essencial à qualidade de vida, como direito fundamental e de uso comum do povo. Isso instrumentaliza normativamente o princípio da prevenção: qualquer atividade que possa ser danosa deve ser evitada, e quando houver dúvida sobre o potencial deletério de uma determinada ação sobre o ambiente, toma-se a decisão mais conservadora, evitando-se a ação. Ao se falar de inovação tecnológica é preciso entender o nível de incerteza ao se tentar criar algo totalmente novo. Para um produto, processo, uma nova ferramenta, a inovação de uma atividade ou a instalação de obra, potencialmente causadora de significativa degradação ao meio ambiente, é necessário o estudo prévio de impacto ambiental, mediante a análise de risco (Brasil, 1988).

Essa proteção ao meio ambiente, constitucionalmente estabelecida, viabiliza a garantia fundamental e difusa do direito à vida, à liberdade e à segurança de todos na instrumentalização da biossegurança, que utiliza um conjunto de normas legais e regulamentares, estabelecendo critérios e técnicas para a manipulação genética, com a finalidade de evitar danos ao meio ambiente e à saúde humana, no contexto amplo da diversidade biológica (Brasil, 2005).

A biossegurança está vinculada à observância da qualidade dos procedimentos e aplicações das técnicas, que objetivam a obtenção de processos, produtos e serviços. Seus verdadeiros fundamentos somente podem ser encontrados por meio de uma ação multidisciplinar que inclua, além das ciências médicas e biológicas, também a filosofia, o direito, a antropologia, a ciência política, a teologia, a comunicação, a sociologia, a economia, dentre outros. Esse mecanismo se dá por meio da sua base conceitual, que parte da utilização de um "conjunto de saberes direcionados para ações de prevenção, minimização ou eliminação de riscos inerentes às atividades de pesquisa, produção, ensino, desenvolvimento tecnológico e prestação de serviços, as 
quais possam comprometer a saúde do Homem, dos animais, das plantas e do ambiente ou a qualidade dos trabalhos desenvolvidos" (CTBio/Fiocruz apud Teixeira; Valle, 1996, p.13).

Tal atribuição conceitual está amplamente ligada ao processo biotecnológico e seu potencial inovador, em especial nos setores da agricultura, da saúde e do ambiente, valorizando a complementariedade entre esses. Somente o debate e o estudo poderão impulsionar ainda mais essa área do conhecimento.

\section{Referências}

BASTOS, V. D. Laboratórios farmacêuticos oficiais e doenças negligenciadas: perspectivas de política pública. Rev BNDES, v.13, n.25, p.269-98, 2006.

BRASIL. Presidência da República. Constituição de 1988. Constituição da República Federativa do Brasil. Brasília: Senado, 1988. 168p.

Lei n.11.105, de 24 de março de 2005. Estabelece normas de segurança e mecanismos de fiscalização de atividades que envolvam organismos geneticamente modificados e seus derivados, cria o Conselho Nacional de Biossegurança, reestrutura a Comissão Técnica Nacional de Biossegurança, dispõe sobre a Política Nacional de Biossegurança. Diário Oficial [da] República Federativa do Brasil, Brasília, DF, p.l, mar. 2005. Seção 1, pt.1.

CASTELLS, M. A Era da Informação: economia, sociedade e cultura. A sociedade em rede. São Paulo: Paz e Terra; 1999. 698p. v.I.

CEZAR, F. G.; ABRANTES, P. C. C. Princípio da precaução: considerações epistemológicas sobre o princípio e sua relação com o processo de análise de risco. Cad Ciência \& Tecnologia, v.20, n.2, p.225-62, 2003.

COSTA, S. B. A negligência em pauta: análise da cobertura jornalística das revistas Veja e Época sobre doenças negligenciadas. 2007. Especialização (monografia em Comunicação Social), Universidade Federal do Maranhão, São Luis, 2007.

DRUGS for Neglected Diseases Initiative - DNDi; 2004. Disponível em: <http:// www.dndi.org.br/Portugues/sobre_dndi.aspx>. Acesso 20 dez. 2011.

EWALD, F. Philosophie politique du principe de précaution. In: EWALD, F.; GOLLIER, C.; SADELEER, N. (Coord.) Le principe de précantion. Paris: PUF, 2001. p.6-72. (Col. "Que sais-je ?")

FOUCAULT, M. Em defesa da sociedade: curso no Collège de France (1975-1976). São Paulo: Martins Fontes; 1999. 134p.

FREITAS, C. M. Avaliação de riscos dos transgênicos orientada pelo princípio da precaução. In: VALLE, S.; TELLES, J. L. (Coord.) Bioética e biorrisco: abordagem transdisciplinar. Rio de Janeiro: Interciência, 2003. p.113-42.

MARQUES, M. B. Acessibilidade aos medicamentos: o desafio de vincular ciência, tecnologia, inovação e saúde no Brasil. Brasília: CGEE, 2002.94p. 
MÉDECINS SANS FRONTIÈRES. MSF/DNDi. Fatal imbalance: the crisis in research and development for drugs for neglected diseases. Genève: Médecins Sans Frontières; 2001. 21p.

MOREL, C. M. A internacionalização de agendas de pesquisa: desafios e perspectivas. Ciência e Cultura, v.57, n.1, p.39-41, 2005.

NAVARRO, M. B. M. A.; CARDOSO, T. A. O. Percepção de risco e cognição: reflexão sobre a sociedade de risco. Ciências \&́ Cognição, v.2, n.6, p.67-72, 2005.

ORGANISATION FOR ECONOMIC CO-OPERATION AND DEVELOPMENT. OCDE. Síntese. Riscos Emergentes no Século XXI: Programa de Ação. Paris: OECD, 2003. 9p.

SCHARAMM, F. R. Introduzindo o problema... In: ENCONTRO DE BIOÉTICA DO CONSELHO REGIONAL DE MEDICINA DO RIO DE JANEIRO (CREMERJ), 5., 2004. A Bioética e o Uso de Células-Tronco. Anais... Recife: CREMERJ, 2004. Disponível em: <http://www.ghente.org/temas/celulas-tronco/ARTIGO\%20 ROLAND_BIOETICA.pdf>. Acesso em: $11 \mathrm{dez} .2011$.

TEIXEIRA, P.; VALLE, S. (Org.) Biossegurança: uma abordagem multidisciplinar. Rio de Janeiro: Ed. Fiocruz; 1996. 362p.

WINNACKER, E. L. Tecnologia genética: intervenções no homem. O que é lícito e o que não é. Cad Adenauer 8: Biotecnologia em discussão, v.1, n.1, p.117-33, 2000.

RESUMO - O artigo reflete sobre os processos construtores de novas tecnologias advindas da dinâmica da produção de novos conhecimentos científicos, que envolvem demandas de mercado, associadas aos interesses industriais, ao consumo e às expectativas sociais diante das inovações tecnológicas, o que impõe à comunidade científica e acadêmica, novos desafios na elaboração de saberes, principalmente, naqueles apoiados na perspectiva da complexidade com a concorrência de múltiplas áreas e campos de conhecimento, incluindo enfaticamente o da biossegurança. Através dessa dinâmica analítica discute-se a essencialidade da biossegurança e sua interface com as inovações tecnológicas e a bioética que se estabelecem pelas vertentes da política em beneficio das ações preventivas, voltadas para promoção da qualidade de processos tecnológicos inovadores direcionados para a saúde.

PALAVRAS-CHAVE: Difusão de inovações, Controle de risco, Saúde pública, Política de Saúde.

ABSTRACT - The present paper places new thoughts on technology building processes originated from the dynamics of scientific knowledge production, involving market demands related to industrial interests, to the consumer and to social expectations face to technological innovation, which implies in new challenges to the scientific and academic community as well as new challenges to the production of thought, leading mainly to a perspective of complexity regarding multiple areas and fields of knowledge, particularly the discipline of Biosafety. Through this analytical dynamics, the essential concepts of Biosafety are discussed with regard to its relationship to Bioethics and technological 
innovation, while being set up by preventive actions and public health policies aimed at the quality of innovative technologic products .

KEYWORDS: Diffusion of innovation, Risk management, Public health, Health policy.

Marli B. M. de Albuquerque Navarro é doutora em História Contemporânea. Pesquisadora sênior do Núcleo de Biossegurança. Escola Nacional de Saúde Pública, Fundação Oswaldo Cruz/Fiocruz. Rio de Janeiro. Brasil. @ - mnavarro@fiocruz.br

Telma Abdalla de Oliveira Cardoso é doutora em Saúde Pública. Pesquisadora sênior do Núcleo de Biossegurança, Escola Nacional de Saúde Pública, Fundação Oswaldo Cruz/Fiocruz. Rio de Janeiro. Brasil. @-abdalla@fiocruz.br

Nery Cunha Vitalé doutor em Ciência Animal. Gerência de Desenvolvimento Científico e Tecnológico, Secretaria de Estado de Saúde de Minas Gerais. Belo Horizonte/ Minas Gerais. Brasil. @- neryvital@gmail.com

Bernardo Elias Correa Soares é doutor em Saúde Pública. Pesquisador sênior do Núcleo de Biossegurança, Escola Nacional de Saúde Pública, Fundação Oswaldo Cruz. Rio de Janeiro. Brasil. @- bernardo@fiocruz.br

Recebido em 17.10.2012 e aceito em 6.11.2012.

I Escola Nacional de Saúde Pública, Fundação Oswaldo Cruz/Fiocruz, Rio de Janeiro/RJ, Brasil.

II Escola Nacional de Saúde Pública, Fundação Oswaldo Cruz/Fiocruz, Rio de Janeiro/RJ, Brasil.

III Secretaria de Estado de Saúde de Minas Gerais, Belo Horizonte/MG, Brasil.

Iv Escola Nacional de Saúde Pública, Fundação Oswaldo Cruz/Fiocruz, Rio de Janeiro/RJ, Brasil. 